\title{
Light-cone Wilson loop in classical lattice gauge theory
}

\section{Laine and A. Rothkopf}

Institute for Theoretical Physics, Albert Einstein Center, University of Bern, Sidlerstrasse 5, CH-3012 Bern, Switzerland

E-mail: laine@itp.unibe.ch, rothkopf@itp.unibe.ch

\begin{abstract}
The transverse broadening of an energetic jet passing through a non-Abelian plasma is believed to be described by the thermal expectation value of a light-cone Wilson loop. In this exploratory study, we measure the light-cone Wilson loop with classical lattice gauge theory simulations. We observe, as suggested by previous studies, that there are strong interactions already at short transverse distances, which may lead to more efficient jet quenching than in leading-order perturbation theory. We also verify that the asymptotics of the Wilson loop do not change qualitatively when crossing the light cone, which supports arguments in the literature that infrared contributions to jet quenching can be studied with dimensionally reduced simulations in the space-like domain. Finally we speculate on possibilities for full four-dimensional lattice studies of the same observable, perhaps by employing shifted boundary conditions in order to simulate ensembles boosted by an imaginary velocity.
\end{abstract}

KeYwords: Thermal Field Theory, Quark-Gluon Plasma, Lattice Gauge Field Theories ARXIV EPRINT: 1304.4443 


\section{Contents}

$\begin{array}{llr}1 & \text { Introduction } & 1\end{array}$

2 General framework 3

3 Analytic expectations 5

3.1 HTL result in continuum 5

$\begin{array}{lll}3.2 & \text { HTL result on a spatial lattice } & 6\end{array}$

$\begin{array}{lll}3.3 & \text { Beyond perturbation theory } & 7\end{array}$

4 Numerical implementation $\quad 9$

5 Conclusions and outlook $\quad 11$

$\begin{array}{ll}\text { A Leading-order perturbative computation in continuum } & 13\end{array}$

$\begin{array}{ll}\text { B Leading-order perturbative computation on a lattice } & 14\end{array}$

\section{Introduction}

When an energetic jet traverses a strongly interacting thermal medium, various interactions take place and lead to dissipation: the jet loses some of its energy and sharpness. The latter phenomenon is referred to as jet broadening, or jet quenching. If its efficiency is measured experimentally as a function of the jet's energy (this can be done particularly well if the total jet momentum is balanced against that of a hard photon, which does not lose energy to the medium [1]), then we may learn something about the properties of the medium itself. The current understanding is that in order to explain the jet quenching observed empirically in heavy ion collision experiments, interactions have to be much stronger than suggested by leading-order perturbation theory (for reviews see, e.g., refs. [2]-[8]).

On an intuitive level, a highly energetic jet can be thought of as a light-cone Wilson line, and the fact that we are probing its fate in the transverse direction leads us to correlate the Wilson line with a slightly displaced Hermitean conjugate. Adding lines at both ends leads to a light-cone Wilson loop. Arguments have been given to make the correspondence precise (see, e.g., refs. [3], [9]-[12]), however it appears difficult to state the form of the error that is made in this approximation. In the following we take the light-cone Wilson loop as a starting point, without dwelling any further on its relation to physically measurable quantities.

In a statistical environment (with a temperature $T$, assumed to be above a few hundred $\mathrm{MeV}$ ), thermal noise leads to decoherence. As a result the light-cone Wilson loop, to be 
denoted by $W$, "decays" at large Minkowskian times $t \gg \hbar / T .{ }^{1}$ Schematically, assuming an appropriate time ordering, we may expect that

$$
\left\langle W\left(t, r_{\perp}\right)\right\rangle_{T} \stackrel{t \gg \hbar / T}{\sim} Z\left(r_{\perp}\right) e^{-i V\left(r_{\perp}\right) t} \sim Z\left(r_{\perp}\right) e^{-i \operatorname{Re} V\left(r_{\perp}\right) t} e^{-\left|\operatorname{Im} V\left(r_{\perp}\right)\right| t}
$$

where $r_{\perp} \equiv\left|\mathbf{r}_{\perp}\right|$ is the length of a 2-dimensional transverse vector; $\operatorname{Re} V\left(r_{\perp}\right)$ is a real phase; and $\langle\ldots\rangle_{T}$ refers to a thermal expectation value. If the coefficient of the exponential decay is represented in Fourier space,

$$
\left|\operatorname{Im} V\left(r_{\perp}\right)\right|=\int_{\mathbf{k}_{\perp}}\left(1-e^{i \mathbf{k}_{\perp} \cdot \mathbf{r}_{\perp}}\right) C\left(k_{\perp}\right),
$$

then $C\left(k_{\perp}\right)$ is often referred to as the "transverse collision kernel" ([13] and references therein). Considering for concreteness a Wilson loop in the fundamental representation, the leading-order expression for $C\left(k_{\perp}\right)$ at small transverse momenta reads ([14], eq. (44))

$$
C\left(k_{\perp}\right)=g^{2} T C_{\mathrm{F}}\left(\frac{1}{k_{\perp}^{2}}-\frac{1}{k_{\perp}^{2}+m_{\mathrm{E}}^{2}}\right)+\mathcal{O}\left(\frac{g^{4} T^{2}}{k_{\perp}^{3}}\right)
$$

where $g^{2} \equiv 4 \pi \alpha_{s} / \hbar$ is the strong gauge coupling; $C_{\mathrm{F}} \equiv\left(N_{\mathrm{c}}^{2}-1\right) /\left(2 N_{\mathrm{c}}\right)$; and

$$
m_{\mathrm{E}}^{2} \equiv\left(\frac{N_{\mathrm{c}}}{3}+\frac{N_{\mathrm{f}}}{6}\right) \frac{g^{2} T^{2}}{\hbar}
$$

is the Debye mass parameter (which has units of inverse distance squared). The question we are interested in is how large the corrections to eq. (1.3) can be, particularly within the infrared domain $k_{\perp} \ll \pi T / \hbar$.

Previous work already exists on infrared corrections to eq. (1.3). In particular, the corrections of $\mathcal{O}\left(g^{4} T^{2}\right)$ were computed for $k_{\perp} \sim m_{\mathrm{E}}$ in ref. [13], and non-perturbative effects of $\mathcal{O}\left(g^{6} T^{3}\right)$ for $k_{\perp} \sim g^{2} T / \pi$ were addressed in ref. [15]. In ref. [13] it was noted that for $k_{\perp} \sim m_{\mathrm{E}}$ the perturbative series might be slowly convergent, and therefore in need of an all-orders resummation. Conceptually, the aim of the current study is to implement such a resummation through numerical simulations of a low-energy description.

More precisely, our goal is to address eq. (1.1) within the framework of classical lattice gauge theory (CLGT). It should be immediately acknowledged that although CLGT does represent ${ }^{2}$ the physics of the system at scales $k_{\perp} \sim g^{2} T / \pi$, it actually is not quantitatively accurate at the scales $k_{\perp} \sim m_{\mathrm{E}}$ that are of most interest here. The reason is that it is highly sensitive to lattice artifacts in this momentum range [23, 24]. Nevertheless, it still contains the correct physics on the qualitative level; indeed CLGT simulations have been useful for gaining insight on various phenomena at the Debye scale (see e.g. refs. [25, 26]), thereby serving as a stepping stone towards full four-dimensional simulations of the same

\footnotetext{
${ }^{1}$ Since the concept of a classical limit appears frequently, it is useful to show $\hbar$ explicitly, thereby keeping the units of time and energy separate. In contrast we set the speed of light equal to unity as usual.

${ }^{2}$ Originally CLGT simulations were employed for addressing the rate of non-perturbative anomalous chirality violation originating from the scale $k_{\perp} \sim g^{2} T / \pi$, see e.g. refs. [16]-[21] and references therein. They have also been used for studying the dynamics of thermal phase transitions, see e.g. ref. [22], as well as many non-equilibrium problems in cosmology and heavy ion collision experiments.
} 


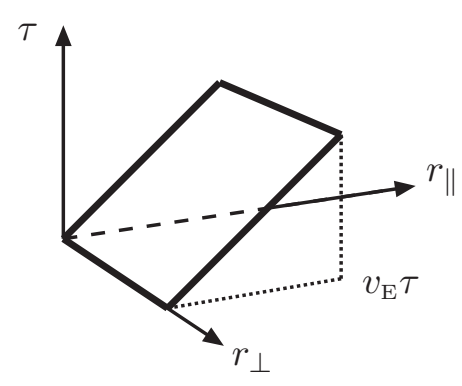

Figure 1. An illustration of a tilted Wilson loop in Euclidean space-time. The slope in the "parallel" direction $\left(r_{\|}\right)$is parametrized by a Euclidean velocity $v_{\mathrm{E}}$, so that the right edge lies at $r_{\|}=v_{\mathrm{E}} \tau$. The transverse extent $\left(r_{\perp}\right)$ can be interpreted as the length of a two-dimensional vector.

problems (see e.g. refs. [27]-[31]). The great strength of CLGT is that it operates directly in Minkowskian space-time, thereby circumventing all issues related to analytic continuation. The purpose of the present study is to explore what CLGT can teach us about the light-cone Wilson loop in the domain indicated in eq. (1.1). ${ }^{3}$

The plan of this paper is the following. After outlining the general framework (section 2), we present some analytic expectations in section 3 , setting the stage for a comparison with numerical data. The numerical results are presented in section 4 , and we conclude in section 5 .

\section{General framework}

With a view on obtaining a formulation which may eventually be amenable to full fourdimensional lattice Monte Carlo simulations, we start by defining a "tilted" Wilson loop in Euclidean space-time. The Wilson loop is parametrized by a transverse extent, $r_{\perp}$; by an imaginary-time variable, $\tau \in(0, \beta)$, where $\beta \equiv \hbar / T$; and by a velocity, $v_{\mathrm{E}}$. At the end of the computation both $\tau$ and $v_{\mathrm{E}}$ will be subjected to a Wick rotation, but for the moment they are treated as real variables. The Wilson loop is illustrated in figure 1. In the limit $v_{\mathrm{E}} \rightarrow 0$, it goes over into the Wilson loop defined in the context of heavy quarkonium physics in ref. [33]. (We note that it may ultimately be more useful to "tilt" the thermal ensemble rather than the Wilson loop, which in four dimensions can be achieved through shifted boundary conditions [34].)

More concretely, starting with the continuum formulation and choosing sign conventions in which the covariant derivative in the fundamental representation is $D_{\mu}=$ $\partial_{\mu}+i g_{0} A_{\mu}$, a straight Wilson line reads

$$
W\left[X_{2} ; X_{1}\right]=\mathcal{P} \exp \left(-i g_{0} \int_{X_{1}}^{X_{2}} \mathrm{~d} X_{\mu} A_{\mu}\right)
$$

where $X \equiv(\tau, \mathbf{x})$. The foremost tilted line of figure 1 can be expressed as

$$
W\left[\left(\tau, \mathbf{r}_{\perp}+\mathbf{v}_{\mathrm{E}} \tau\right) ;\left(0, \mathbf{r}_{\perp}\right)\right]=\mathbb{1}-i g_{0} \int_{0}^{\tau} \mathrm{d} \tau_{1}\left(A_{0}+\mathbf{v}_{\mathrm{E}} \cdot \mathbf{A}\right)\left(\tau_{1}, \mathbf{r}_{\perp}+\mathbf{v}_{\mathrm{E}} \tau_{1}\right)+\ldots,
$$

\footnotetext{
${ }^{3}$ Previously CLGT simulations have been used as an ingredient in a phenomenological study of jet quenching of hard particles [32], but the light-cone Wilson loop was not measured.
} 
where $\mathbf{v}_{\mathrm{E}} \equiv v_{\mathrm{E}} \mathbf{e}_{\|}$. The expectation value of the Wilson loop is defined as

$$
C_{\mathrm{E}}\left(\tau, v_{\mathrm{E}}, r_{\perp}\right) \equiv \frac{1}{N_{\mathrm{c}}} \operatorname{Tr}\left\langle W\left[(0, \mathbf{0}) ;\left(\tau, \mathbf{v}_{\mathrm{E}} \tau\right) ;\left(\tau, \mathbf{r}_{\perp}+\mathbf{v}_{\mathrm{E}} \tau\right) ;\left(0, \mathbf{r}_{\perp}\right) ;(0, \mathbf{0})\right]\right\rangle_{T},
$$

where the thermal average $\langle\ldots\rangle_{T}$ implies periodic boundary conditions for bosonic and antiperiodic ones for fermionic fields over the Euclidean time direction. In the following, we have in mind evaluating the expectation value within pure $\mathrm{SU}(3)$ gauge theory, even though this restriction can in principle be relaxed.

Since the physical observable that we are interested in refers to Minkowskian time, an analytic continuation needs to be carried out at the end of the computation. Technically, we do this by substituting $\tau \rightarrow i t$, which for 2-point functions yields the time ordering corresponding to a Wightman correlator denoted by $C_{>}$. (The Wilson loop can always be thought of as a 2-point function in time if the tilted lines are gauged to unity; general issues related to time ordering have been discussed in refs. [3], [9]-[13].) However, since in the following we will simultaneously take the classical limit, time ordering actually plays no role. The classical limit can be defined by writing

$$
\beta=\frac{\hbar}{T}, \quad g_{0}^{2}=g^{2} \hbar,
$$

and subsequently setting $\hbar \rightarrow 0$ [35]. This limit is non-trivial and results in an interacting non-Abelian gauge theory which captures the infrared features of the system's real-time thermal dynamics $[16,17]$.

Apart from the continuum formulation, we also consider a lattice formulation of the theory in the following. Like in ref. [36], the theory is discretized only in spatial directions, with a finite lattice spacing $a$, whereas the time direction remains continuous. ${ }^{4}$ Thereby the four-dimensional Euclidean action can formally be expressed as

$$
S_{\mathrm{E}} \equiv a^{3} \sum_{\mathbf{x}} \int_{0}^{\beta} \mathrm{d} \tau\left\{\sum_{i=1}^{3} \operatorname{Tr}\left[E_{i}^{2}(X)\right]+\frac{1}{a^{4} g_{0}^{2}} \sum_{i, j=1}^{3} \operatorname{Tr}\left[\mathbb{1}-P_{i j}(X)\right]\right\},
$$

where $g_{0}$ denotes the bare gauge coupling and $E_{i}, P_{i j}$ denote the electric field strength and the spatial plaquette, respectively:

$$
\begin{aligned}
E_{i}(X) & \equiv-\frac{i\left[\partial_{\tau} U_{i}(X)\right] U_{i}^{\dagger}(X)}{a g_{0}}+\frac{A_{0}(X)-U_{i}(X) A_{0}\left(X+a \mathbf{e}_{i}\right) U_{i}^{\dagger}(X)}{a}, \\
P_{i j}(X) & \equiv U_{i}(X) U_{j}\left(X+a \mathbf{e}_{i}\right) U_{i}^{\dagger}\left(X+a \mathbf{e}_{j}\right) U_{j}^{\dagger}(X) .
\end{aligned}
$$

Here $U_{i} \in \mathrm{SU}(3)$ are link matrices, and $A_{0}$ is a traceless and Hermitean gauge field. The action is invariant under the gauge transformation

$$
\begin{aligned}
& U_{i}(X) \rightarrow G(X) U_{i}(X) G^{-1}\left(X+a \mathbf{e}_{i}\right), \\
& A_{0}(X) \rightarrow G(X) A_{0}(X) G^{-1}(X)+\frac{i}{g_{0}}\left[\partial_{\tau} G(X)\right] G^{-1}(X),
\end{aligned}
$$

\footnotetext{
${ }^{4}$ This formulation is invoked because of its close relation to CLGT; in contrast, the speculations to be made about full four-dimensional lattice studies in section 5 apply equally well to the standard formulation with a symmetric discretization in all directions.
} 
with $G \in \mathrm{SU}(3)$. For perturbative computations we make use of covariant gauges; in contrast, on the real-time simulation side it is convenient to make use of the corresponding Hamiltonian formulation with a vanishing Minkowskian $A_{0}$ and a corresponding Gauss law constraint.

\section{Analytic expectations}

\subsection{HTL result in continuum}

Our ultimate goal is to compute the analytic continuation of eq. (2.3) at large Minkowskian times, $t \gtrsim \pi /\left(g^{2} T\right)$, and large transverse distances, $r_{\perp} \gtrsim 1 / m_{\mathrm{E}}$. We start, however, by inspecting short distances, $r_{\perp} \lesssim 1 / m_{\mathrm{E}}$. This can be done with perturbation theory, provided that we recall that at high temperatures the loop expansion needs to be resummed to all orders in order to arrive at a consistent weak-coupling result. We are working at leading non-trivial order in this regime, and then the effects of resummation are contained within Hard Thermal Loop (HTL) [37, 38] propagators.

Concretely, we carry out the computation by evaluating the graphs of figure 2 with the Euclidean propagator

$$
\left\langle A_{\mu}^{a}(X) A_{\nu}^{b}(Y)\right\rangle=\delta^{a b} \sum_{K} e^{i K \cdot(X-Y)}\left[\frac{\mathbb{P}_{\mu \nu}^{\mathrm{T}}(K)}{K^{2}+\Pi_{\mathrm{T}}}+\frac{\mathbb{P}_{\mu \nu}^{\mathrm{E}}(K)}{K^{2}+\Pi_{\mathrm{E}}}+\frac{\xi K_{\mu} K_{\nu}}{K^{4}}\right],
$$

where $K \equiv\left(k_{n}, \mathbf{k}\right)$ and $\xi$ is a gauge parameter. The projectors read

$$
\mathbb{P}_{\mu \nu}^{\mathrm{T}}(K)=\delta_{\mu i} \delta_{\nu j}\left(\delta_{i j}-\frac{k_{i} k_{j}}{k^{2}}\right), \quad \mathbb{P}_{\mu \nu}^{\mathrm{E}}(K)=\delta_{\mu \nu}-\frac{K_{\mu} K_{\nu}}{K^{2}}-\mathbb{P}_{\mu \nu}^{\mathrm{T}}(K) .
$$

The Euclidean propagators are expressed in a spectral representation,

$$
\frac{1}{K^{2}+\Pi_{\mathrm{T}(\mathrm{E})}}=\int_{-\infty}^{\infty} \frac{\mathrm{d} k_{0}}{\pi} \frac{\rho_{\mathrm{T}(\mathrm{E})}(\mathcal{K})}{k_{0}-i k_{n}}
$$

where $\mathcal{K} \equiv\left(k_{0}, \mathbf{k}\right)$, and subsequently the Matsubara sums are carried out. The explicit forms of the self-energies can be found in the literature but are not needed here. In general the computation parallels that in ref. [33], except that it is in some sense simpler (as long as we stay in continuum): indeed a non-zero $v_{\mathrm{E}}$ "regulates" the contributions of the Matsubara zero modes, so that they no longer need to be treated separately from the non-zero ones.

Some technical details of the computation are presented in appendix A. Here we merely note that analytic continuation is carried out as $\tau \rightarrow i t, v_{\mathrm{E}} \rightarrow-i v$, and the classical limit is taken as discussed around eq. (2.4). ${ }^{5}$ The definition of a potential reads (cf. eq. (1.1))

$$
i \partial_{t} C_{\mathrm{E}}\left(i t,-i v, r_{\perp}\right) \equiv V\left(t, v, r_{\perp}\right) C_{\mathrm{E}}\left(i t,-i v, r_{\perp}\right)
$$

\footnotetext{
${ }^{5}$ In practice the classical limit amounts to assuming that $k_{0} \ll \pi T / \hbar$; therefore, at leading order it correctly represents the physics of the large-time or low-energy limit of the exponential decay.
} 


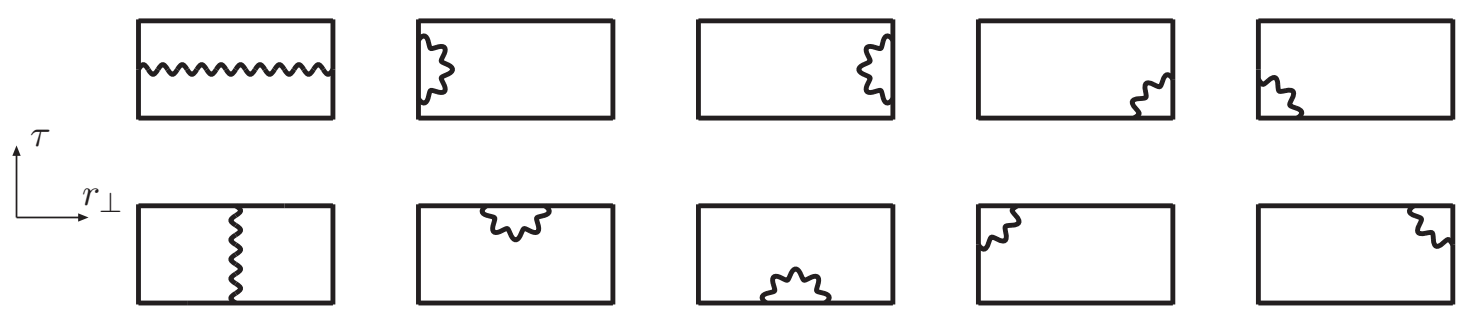

Figure 2. The graphs contributing to the tilted Wilson loop of figure 1 at $\mathcal{O}\left(g_{0}^{2}\right)$. Wiggly lines stand for HTL-resummed gluon propagators.

taking the limit $t \rightarrow \infty$ and setting $v \rightarrow 1$, we reproduce the result of eq. (1.3):

$$
\begin{aligned}
V_{\mathrm{cl}}^{(2)}\left(\infty, 1, r_{\perp}\right) & =-i g^{2} T C_{\mathrm{F}} \int_{\mathbf{k}_{\perp}}\left(1-\cos \mathbf{k}_{\perp} \cdot \mathbf{r}_{\perp}\right)\left(\frac{1}{k_{\perp}^{2}}-\frac{1}{k_{\perp}^{2}+m_{\mathrm{E}}^{2}}\right) \\
& =-i \frac{g^{2} T C_{\mathrm{F}}}{2 \pi}\left[\ln \left(\frac{m_{\mathrm{E}} r_{\perp}}{2}\right)+\gamma_{\mathrm{E}}+K_{0}\left(m_{\mathrm{E}} r_{\perp}\right)\right] .
\end{aligned}
$$

Here $K_{0}$ is a modified Bessel function.

Next-to-leading order (NLO) corrections to the integrand of eq. (3.5) have been determined in ref. [13]. They are large and increase the magnitude of the imaginary part; their numerical contribution to eq. (3.6) is shown in figure 4 below.

\subsection{HTL result on a spatial lattice}

For a practical measurement, the theory needs to be regularized; within CLGT, this means that we consider (a Minkowski-space classical limit of) the theory defined by eq. (2.5). Expressing everything in lattice units and taking the limit of eq. (2.4), the results depend on a single parameter, which we denote by

$$
\beta_{\mathrm{G}} \equiv \frac{2 N_{\mathrm{c}}}{g^{2} T a} .
$$

Initial configurations are generated with the weight $\exp \left(-\beta_{\mathrm{G}} H_{\mathrm{cl}}\right) \prod_{\mathbf{x}} \delta(\mathcal{G}(\mathbf{x}))$, where

$$
H_{\mathrm{cl}}=\sum_{\mathbf{x}}\left\{\sum_{i=1}^{3} \operatorname{Tr}\left[\mathcal{E}_{i}^{2}(\mathbf{x})\right]+\frac{1}{2 N_{\mathrm{c}}} \sum_{i, j=1}^{3} \operatorname{Tr}\left[\mathbb{1}-P_{i j}(\mathbf{x})\right]\right\} ;
$$

$\mathcal{G}(\mathbf{x})$ denotes the Gauss law constraint; and $\mathcal{E}_{i}(\mathbf{x})$ are suitably normalized canonical momenta conjugate to the link matrices $U_{i}(\mathbf{x})$. Subsequently the fields are evolved according to classical equations of motion (cf. eqs. (4.1), (4.2)), and the observable is measured as illustrated in figure 3. (Further details on CLGT simulations can be found e.g. in refs. [17]$[20],[25,26]$; the normalization of the electric field is strongly reference-dependent.)

Within CLGT, the Debye mass scale of the continuum formulation gets replaced with $m_{\mathrm{E}}^{2} \rightarrow g^{2} T / a$, whereas the coupling constant scale remains put at $g^{2} T$. In lattice units, this implies that we want to determine the Wilson loop at separations $r_{\perp} / a \gtrsim \sqrt{\beta_{\mathrm{G}}}$ and time scales $t / a \gtrsim \beta_{\mathrm{G}}$. The latter of these requirements poses a significant challenge at large $\beta_{\mathrm{G}}$, and introduces a source of systematic errors with any limited resources. Approaching this 


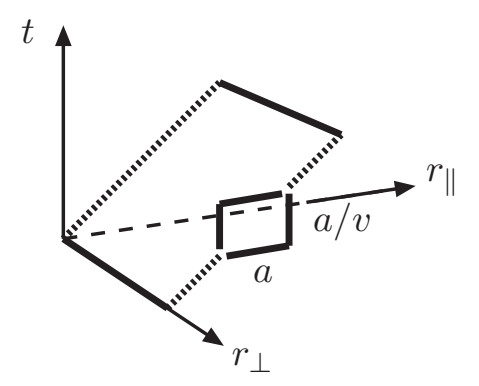

Figure 3. An illustration of a tilted Wilson loop after the discretization of the spatial directions with a lattice spacing $a$, and a Wick rotation of both $\tau$ and $v_{\mathrm{E}}$ to Minkowskian space-time. We represent the tilted Wilson lines by averaging the smallest possible building blocks over the upper and lower paths. Measurements are taken at values $t=n a / v$, with $n \in \mathbb{N}$.

regime from below, perturbation theory can again be used, but necessitates a HTL-type resummation, whose details were worked out in refs. [23, 24].

In practice, carrying out perturbative computations even to leading non-trivial order is cumbersome, due to the asymmetry in the discretizations of the temporal and spatial directions. As an example, the expression obtained after carrying out the Wick contractions for the graphs in figure 2 is shown in appendix B. As a main qualitative difference with respect to the continuum computation, we note that the tilted Wilson lines in figure 3 do not cancel against each other even at distance $r_{\perp}=0$. Rather, we obtain an "intercept" which we denote by

$$
\mathcal{I}(v) \equiv \frac{2 v a}{3} \int_{\mathbf{k}} \frac{\sin ^{2}\left(\frac{a \tilde{k}}{2 v}\right)}{\tilde{k}^{2}}, \quad \tilde{k} \equiv \sqrt{\tilde{k}^{2}}, \quad \tilde{k}^{2} \equiv \sum_{i=1}^{3} \tilde{k}_{i}^{2}
$$

where $\int_{\mathbf{k}}$ and $\tilde{k}_{i}$ are defined in eq. (B.1). Then we expect eq. (3.5) to be replaced through

$$
V_{\mathrm{cl}}^{(2)}\left(\infty, 1, r_{\perp}\right) \simeq-i g^{2} T C_{\mathrm{F}}\left\{\mathcal{I}(1)+\int_{\mathbf{k}_{\perp}}\left(1-\cos k_{y} r_{\perp}\right)\left(\frac{1}{\tilde{k}_{y}^{2}+\tilde{k}_{z}^{2}}-\frac{1}{\tilde{k}_{y}^{2}+\tilde{k}_{z}^{2}+m_{\mathrm{E}}^{2}}\right)\right\}
$$

where $\mathbf{k}_{\perp} \equiv\left(k_{y}, k_{z}\right)$, and the Debye mass parameter reads [23, 24, 39, 40]

$$
m_{\mathrm{E}}^{2}=2 g^{2} T N_{\mathrm{c}} \frac{\Sigma}{4 \pi a}, \quad \Sigma=\Gamma^{2}\left[\frac{1}{24}\right] \Gamma^{2}\left[\frac{11}{24}\right] \frac{\sqrt{3}-1}{48 \pi^{2}} .
$$

As discussed in appendix B, the $r_{\perp}$-dependent part of eq. (3.10) is an approximation, but is expected to be valid for $r_{\perp} \gg a$. In any case eq. (3.10) illustrates the general feature that, apart from the scale of the lattice spacing, the potential can have non-trivial structure only at two distance scales, namely $1 / g^{2} T$ and $1 / m_{\mathrm{E}}$.

\subsection{Beyond perturbation theory}

Let us extract lessons from above for what we may expect to see in the simulations:

- At "short" distances, $r_{\perp} \ll 1 / m_{\mathrm{E}}, \operatorname{Im} V_{\mathrm{cl}}$ should start off with a non-zero intercept, given by eq. (3.9) for large $\beta_{\mathrm{G}}$. 

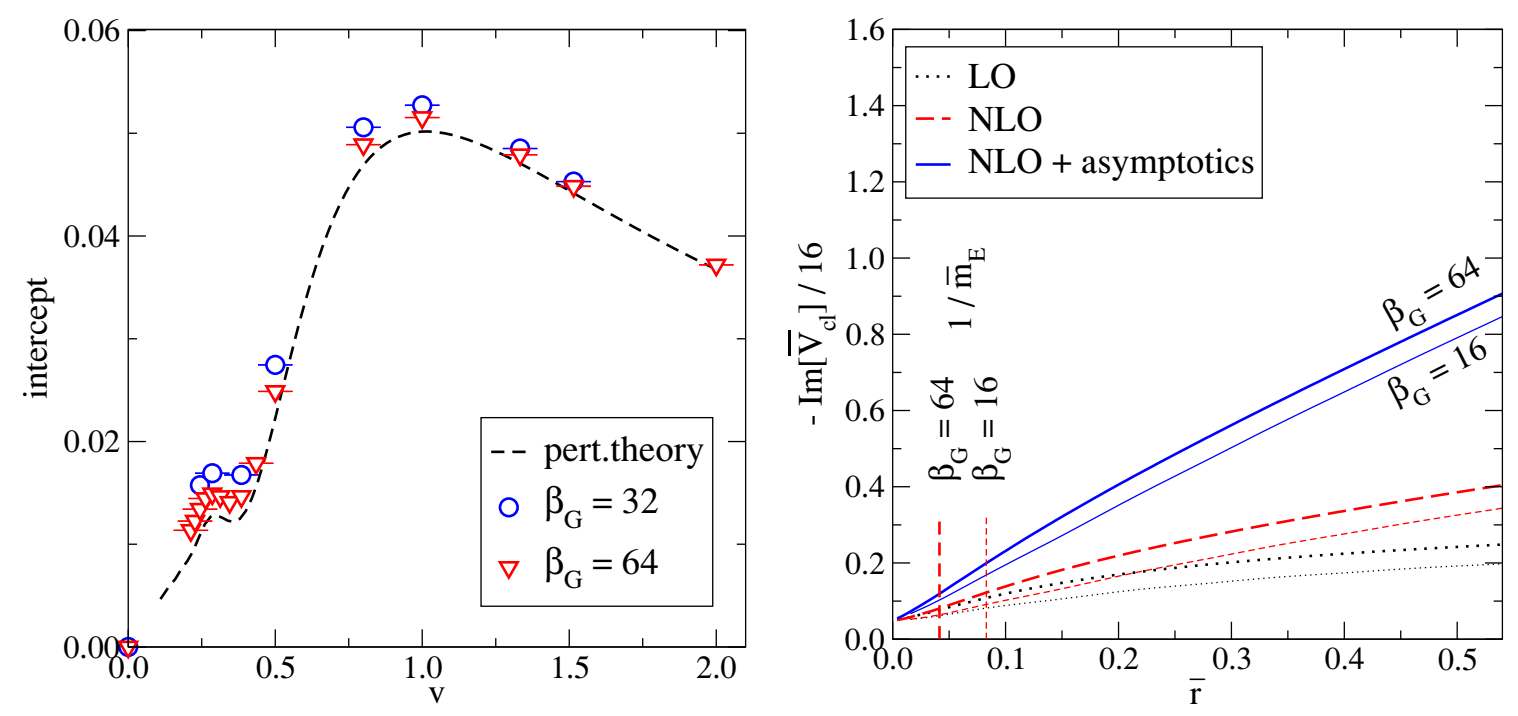

Figure 4. Left: the intercept from eq. (3.9), in units of eq. (3.15), compared with lattice data. Right: the potential from eq. (3.10) ("LO"); with an integrand taken from ref. [13] ("NLO"); and after adding the long-distance asymptotics [15] ("NLO+asymptotics"). Vertical lines indicate distances beyond which perturbation theory is unreliable. The asymptotic behaviour sets in at $\bar{r} \gtrsim 2 r_{0} g^{2} T /\left(2 N_{\mathrm{c}}\right) \approx 0.73$. Axis ranges have been chosen to agree with figure 7 (left) in which lattice data is shown.

- At "intermediate" distances, $r_{\perp} \sim 1 / m_{\mathrm{E}}$, the potential $\operatorname{Im} V_{\mathrm{cl}}$ shows a non-trivial structure which is relevant for jet quenching. This structure cannot be studied quantitatively with the approach of the present paper, given that within CLGT the Debye scale is completely determined by lattice artifacts, cf. eq. (3.11). On the qualitative level, however, we expect large corrections to the leading-order expression in eq. (3.10) [13].

- At "long" distances, $r_{\perp} \gg 1 / m_{\mathrm{E}}$, the phenomena related to the Debye scale are exponentially screened, and the physics is dominated by the colour-magnetic scale $g^{2} T / \pi$. More precisely, in continuum the imaginary part of the light-cone potential corresponds to the static potential of three-dimensional pure Yang-Mills theory [15], which for $r_{\perp} \gg \pi /\left(g^{2} T\right)$ evaluates to $\left|\operatorname{Im} V_{\mathrm{cl}}\right| \simeq 0.553\left(g^{2} T\right)^{2} r_{\perp}$ for $N_{\mathrm{c}}=3$ [41]. When summed together with the NLO result from ref. [13], which already includes a part of the linear term, the appropriate correction reads $\delta\left|\operatorname{Im} V_{\mathrm{cl}}\right| \simeq[0.553-$ $7 /(16 \pi)]\left(g^{2} T\right)^{2} r_{\perp}[15]$.

In order to observe the features mentioned in the data, it is helpful to change units. Suppose that we use $\beta_{\mathrm{G}}$ from eq. (3.7) in order to convert lattice units to physical units. Then we can express distances and the potential as

$$
\begin{aligned}
\bar{r} & \equiv \frac{r_{\perp} g^{2} T}{2 N_{\mathrm{c}}}=\frac{r_{\perp}}{a \beta_{\mathrm{G}}}, \\
\frac{\operatorname{Im} \bar{V}_{\mathrm{cl}}}{16} & \equiv \frac{1}{16} \frac{2 N_{\mathrm{c}} \operatorname{Im} V_{\mathrm{cl}}}{g^{2} T}=\frac{\beta_{\mathrm{G}} \operatorname{Im} a V_{\mathrm{cl}}}{16},
\end{aligned}
$$



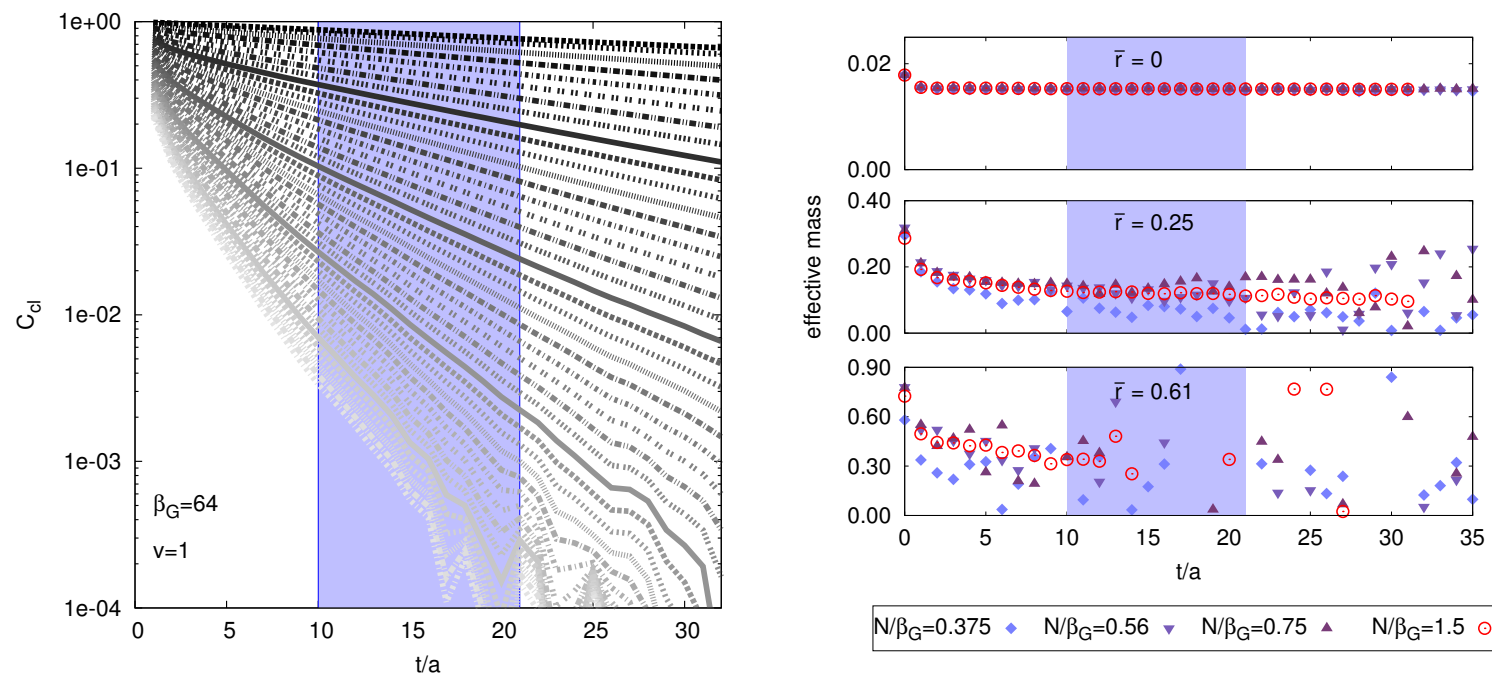

$\mathrm{N} / \beta_{\mathrm{G}}=0.375 \vee \mathrm{N} / \beta_{\mathrm{G}}=0.56 \vee \mathrm{N} / \beta_{\mathrm{G}}=0.75 \wedge \mathrm{N} / \beta_{\mathrm{G}}=1.5 \odot$

Figure 5. Left: time dependence of the classical Wilson loop for $v=1$ at different distances $\bar{r}$. The common fitting range for the determination of $\operatorname{Im} V_{\mathrm{cl}}$ is denoted by the shaded region. Right: effective mass plots for three selected $\bar{r}$. The fitting range $t / a \in[10,20]$ is determined such that a satisfactory signal-to-noise ratio is obtained, however systematic errors could be substantial and the results obtained should be thought of as upper bounds as usual.

where the factor 16 is a convention. In these units, the Debye scale corresponds to

$$
\frac{1}{\bar{m}_{\mathrm{E}}} \equiv \frac{g^{2} T}{2 N_{\mathrm{c}} m_{\mathrm{E}}}=\sqrt{\frac{\pi}{\beta_{\mathrm{G}} \Sigma N_{\mathrm{c}}^{2}}},
$$

the zero-distance intercept from eq. (3.10) amounts to

$$
\lim _{\bar{r} \ll 1 / \bar{m}_{\mathrm{E}}} \frac{\left|\operatorname{Im} \bar{V}_{\mathrm{cl}}\right|}{16}=\frac{2 N_{\mathrm{c}} C_{\mathrm{F}} \mathcal{I}(1)}{16}=\frac{\mathcal{I}(1)}{2},
$$

whereas the long-distance asymptotics reads

$$
\lim _{\bar{r} \gg 1 / \bar{m}_{\mathrm{E}}} \frac{\left|\operatorname{Im} \bar{V}_{\mathrm{cl}}\right|}{16} \approx \frac{1}{16} 0.553\left(2 N_{\mathrm{c}}\right)^{2} \bar{r} \approx 1.2 \bar{r}
$$

The various features together with the effect of NLO corrections [13] are illustrated in figure 4 . The scale $1 / \bar{m}_{\mathrm{E}}$ defines the point beyond which perturbation theory is no longer to be trusted, and the result may eventually (for $\bar{r} \gtrsim 1$ ) go over into the "asymptotics" curve reflecting non-perturbative colour-magnetic dynamics. The non-perturbative contribution of the scales $k_{\perp} \sim g^{2} T / \pi$ to the so-called jet quenching parameter, $\hat{q}$, is however determined by distances just above $r_{\perp} \sim 1 / m_{\mathrm{E}}$, rather than by the long-distance asymptotics [15].

\section{Numerical implementation}

The simulations in the CLGT formalism are carried out along the lines of ref. [25], in which the case $v=0$ was considered. With the choice of temporal gauge $U_{0}(\mathbf{x}, t)=1$ the classical 

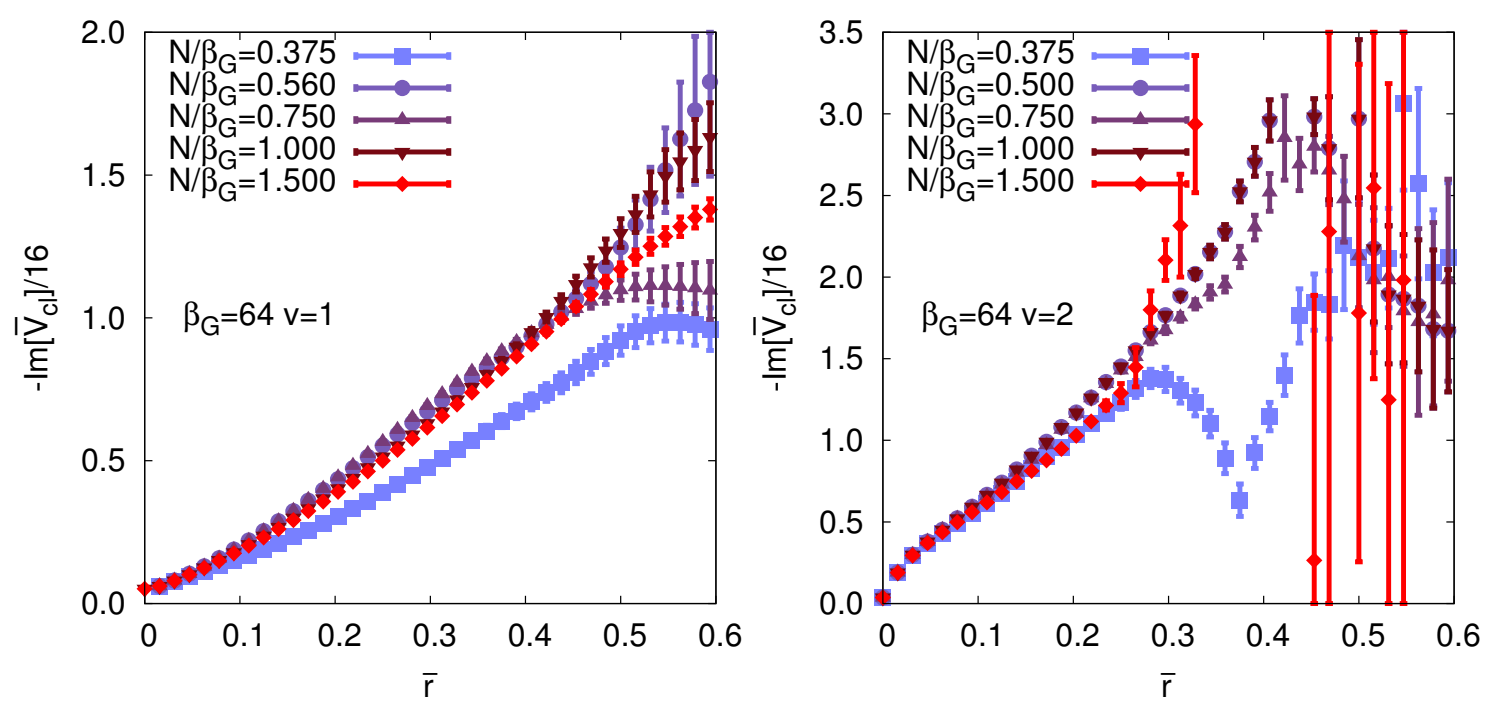

Figure 6. Volume dependence of the imaginary part of the potential as obtained from lattice simulations with $\beta_{\mathrm{G}}=64, v=1$ (left) and $v=2$ (right), and a fitting procedure as described in figure 5 .
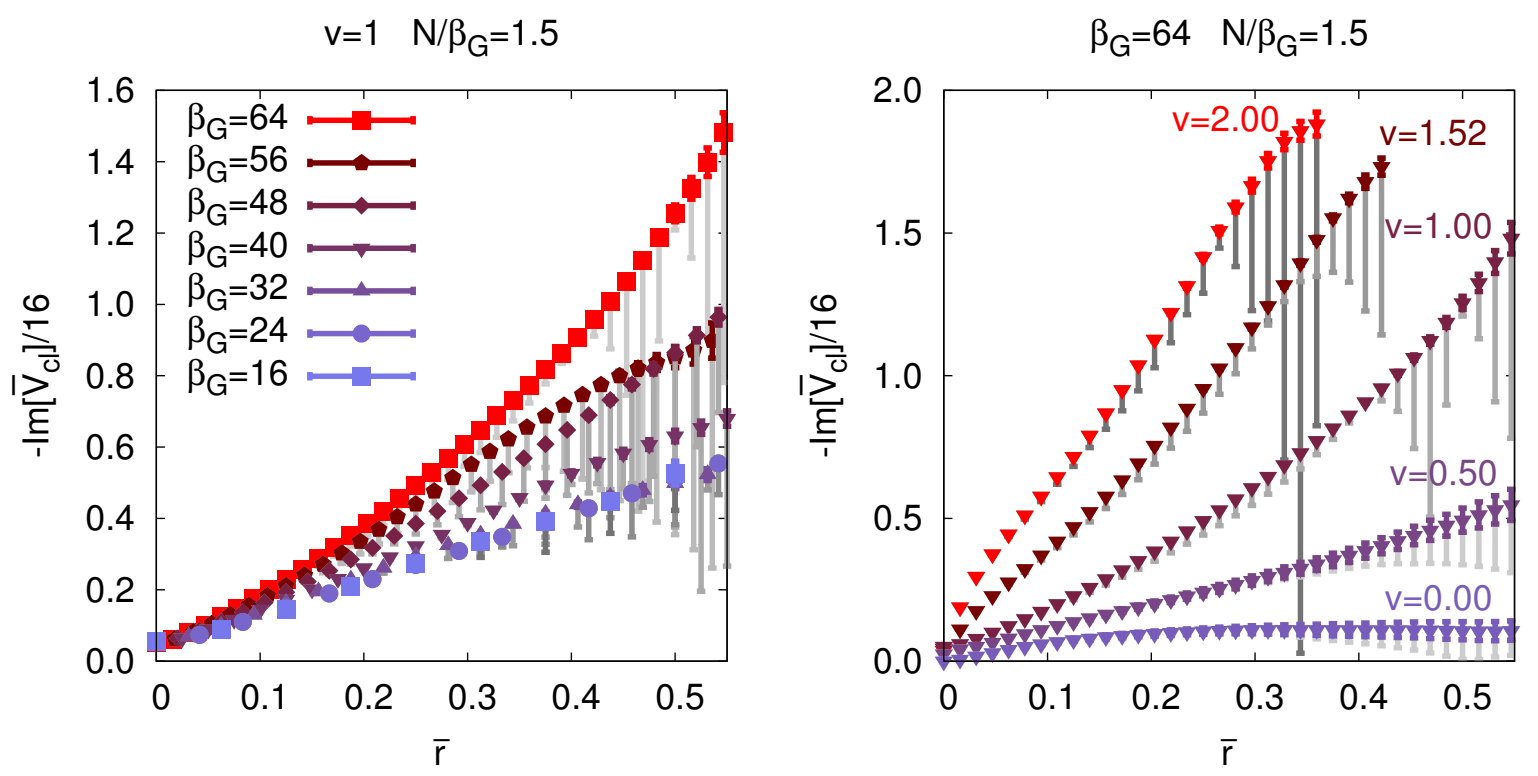

Figure 7. Left: the $\beta_{\mathrm{G}}$ dependence of the imaginary part of the potential for $v=1$ at a fixed physical volume $N / \beta_{\mathrm{G}}=1.5$. Right: velocity dependence for $\beta_{\mathrm{G}}=64$ and $N / \beta_{\mathrm{G}}=1.5$. Coloured bars denote statistical errors, whereas systematic errors, estimated from pushing the fitting ranges to larger $t / a$, are given in gray (fitting at later times always decreases the result).

equations of motion for the variables of eq. (3.8) read

$$
\begin{aligned}
& a \partial_{t} U_{i}(\mathbf{x}, t)=i\left(2 N_{\mathrm{c}}\right)^{\frac{1}{2}} \mathcal{E}_{i}(\mathbf{x}, t) U_{i}(\mathbf{x}, t), \\
& a \partial_{t} \mathcal{E}_{i}^{b}(\mathbf{x}, t)=-\left(\frac{2}{N_{\mathrm{c}}}\right)^{\frac{1}{2}} \operatorname{Im} \operatorname{Tr}\left[T^{b} U_{i}(\mathbf{x}, t) \sum_{|j| \neq i} S_{i j}^{\dagger}(\mathbf{x}, t)\right],
\end{aligned}
$$


where $S_{i j}$ denotes a staple. These differential equations are solved on a hypercubic threedimensional spatial lattice of size $N^{3}$ using the Euler forward finite-difference scheme with temporal lattice spacing $a_{t}=\frac{a}{100}$. As initial conditions, we deploy field configurations thermalized according to refs. $[17-19,25]$ with the Hamiltonian of eq. (3.8) and the appropriate projection to the hypersurface respecting the Gauss law.

To obtain the potential of eq. (3.4), we measure the discretized Wilson loop (defined like in eq. (2.3) but in Minkowski signature) in real time for several different transverse separations $\bar{r}$. The tilting away from the temporal axis with velocity $v$ is implemented as indicated in figure 3. Fitting the time evolution of these purely real quantities (left panel of figure 5) with an exponential allows us to read off $\operatorname{Im} V_{\mathrm{cl}}$ from the exponent. For a rough estimate of $\lim _{t \rightarrow \infty} \operatorname{Im} V_{\mathrm{cl}}$, we identify a common fitting range for all values of $\bar{r}$, in which the asymptotic exponential falloff appears to have stabilized, while at the same time the statistical noise due to a finite number of measurements is still relatively small (right panel of figure 5). These requirements are hard to satisfy for large $\bar{r}$ and $\beta_{\mathrm{G}}$ and, as can also be deduced from figure 5 (right) by bare eye, the procedure adopted is likely to lead to an overestimate of $\lim _{t \rightarrow \infty} \operatorname{Im} V_{\mathrm{cl}}$.

The effect of a finite volume on the determination of $\operatorname{Im} V_{\mathrm{cl}}$ is shown in figure 6 for $v=1$ and $v=2$. Higher velocities lead to a faster exponential damping of the Wilson loop, hence the region for an exponential fit shrinks and leads to a more noisy signal as shown in the right panel. We find that to go to $\bar{r} \simeq 0.4$ a lattice extent of at least $N \gtrsim 1.5 \beta_{\mathrm{G}}$ is necessary.

Once an adequate lattice extent and a usable fitting range $t / a \in[10,20]$ have been established, we proceed to measure the velocity dependence of $\operatorname{Im} V_{\mathrm{cl}}$ and its intercept. For $\bar{r} \lesssim 1 / \bar{m}_{\mathrm{E}}$ perturbation theory becomes more accurate at larger $\beta_{\mathrm{G}}$, and indeed the lattice results approach the perturbative ones for the intercept at all velocities, cf. figure 4(left).

For $\bar{r} \gtrsim 1 / \bar{m}_{\mathrm{E}}$, in contrast, perturbation theory need not be accurate. It is perhaps surprising then how well the "NLO+asymptotics" result works for moderate $\beta_{\mathrm{G}}$, cf. figures 4(right) and 7 (left), even though discrepancies remain at the smallest and largest $\beta_{\mathrm{G}}$ 's. For the smallest $\beta_{\mathrm{G}}$ 's this may be due to the fact that the Debye scale is larger [cf. figure 4 (right)] and therefore the asymptotics is approached at larger separations. For the largest $\beta_{\mathrm{G}}$ we reiterate that it is difficult to reach the regime $t / a \gtrsim \beta_{\mathrm{G}}$ needed for extracting the correct asymptotics (cf. figure 5), so that the remaining discrepancy is probably due to systematic errors. Comparisons with perturbation theory need to be refined with other methods in the Euclidean domain [13], in which the Debye scale is free from lattice artifacts, so that the infinite volume and continuum limits can be systematically taken.

\section{Conclusions and outlook}

The purpose of this exploratory study has been to probe the contribution that highly occupied classical gauge fields make to the thermal expectation value of a light-cone Wilson loop. We have observed that discrepancies to the leading-order expression set in already at relatively short transverse distances, and lead to a larger magnitude of the imaginary part of the potential (stronger interactions) than predicted by leading-order perturbation 
theory (cf. figure 4(right) vs. figure 7(left)). This is in qualitative agreement with the NLO computation of ref. [13] and with the long-distance asymptotics as analyzed in ref. [15]. Quantitative comparisons are hard because of discretization artifacts inherent to the CLGT framework.

In addition, we have noted that crossing the light cone does not change the structure of the potential in any qualitative way (cf. figure 7(right)). This poses well for the proposal of ref. [13] according to which the potential could be measured within a purely static dimensionally reduced effective field theory $[42,43]$. Unlike classical lattice gauge theory, that framework is (super)renormalizable, so that divergences and discretization artifacts can be handled through local counterterms and analytic computations, and the genuine continuum physics of the momentum scale $k_{\perp} \sim m_{\mathrm{E}}$ can be disentangled. (It is useful to stress again that asymptotically large values of $r_{\perp}$ need not be studied [15].) Thereby the existence of large infrared effects contributing to jet quenching can possibly be confirmed, perhaps leading to a QCD-based explanation for the experimentally observed efficient jet quenching in current heavy ion collision experiments at the LHC.

We would finally like to pose the question of whether the observable of eq. (2.3) can also be addressed with direct four-dimensional lattice simulations. One lesson from our study is that discretizing the tilted Wilson lines (cf. figure 3) is inconvenient. It might rather be sensible to boost the ensemble by making use of shifted boundary conditions [34], and measure the Wilson loop always along the time-like lattice direction.

Of course, measuring eq. (2.3) is not enough, but subsequently analytic continuations are needed for extracting the proper real-time physics. In fact there are two separate analytic continuations here: $\tau \rightarrow i t$ as well as $v_{\mathrm{E}} \rightarrow-i v$. The former is conventionally implemented by going through frequency space, i.e. estimating the spectral function corresponding to the Euclidean correlator; from the spectral function, any time ordering can be recovered. For $v_{\mathrm{E}}=0$, a determination of the spectral function has been attempted $[27,44,45]$, and even though systematic uncertainties remain difficult to quantify, the challenge should not be much harder in the presence of $v_{\mathrm{E}} \neq 0$. Note that the quantity of interest here corresponds to the imaginary part of the real-time potential, cf. eqs. (1.1), $(1.2)$.

As far as the analytic continuation of the velocity is concerned, one of the methods used in studies of QCD with a baryonic chemical potential might turn out to be helpful. For instance, one could first carry out simulations with $v_{\mathrm{E}}$; fit the results to a Taylor series; and subsequently carry out an analytic continuation. Although in a mathematical sense a singularity cannot be excluded as $v \rightarrow 1$, we have not observed any drastic changes in the infrared dynamics of the system in this limit (the discretization-specific wobbles around $v \sim 0.3$ in figure 4 (left) are not expected to be present if a boosted ensemble is simulated). Therefore it is conceivable that such a procedure could yield at least qualitative results against which dimensionally reduced simulations, carried out on the space-like side of the light cone, can be compared. 


\section{Acknowledgments}

This work was partly supported by the Swiss National Science Foundation (SNF) under grant 200021-140234 and by the European Commission under the FP7 programme HadronPhysics3.

\section{A Leading-order perturbative computation in continuum}

We compute the graphs in figure 2 with the propagator of eq. (3.1), first in Euclidean space-time. Carrying out Wick contractions, it can be checked that any gauge parameter dependence cancels. Inserting eq. (3.3) for $1 /\left(K^{2}+\Pi_{\mathrm{T}(\mathrm{E})}\right)$, the remaining expression reads

$$
\begin{aligned}
C_{\mathrm{E}}^{(0)}\left(\tau, v_{\mathrm{E}}, r_{\perp}\right)= & 1, \\
C_{\mathrm{E}}^{(2)}\left(\tau, v_{\mathrm{E}}, r_{\perp}\right)= & \frac{g_{0}^{2} C_{\mathrm{F}}}{\beta} \int_{\mathbf{k}}\left(\cos \mathbf{k} \cdot \mathbf{r}_{\perp}-1\right) \int_{-\infty}^{\infty} \frac{\mathrm{d} k_{0}}{\pi} \sum_{k_{n}} \frac{2-e^{i\left(k_{n}+\mathbf{k} \cdot \mathbf{v}_{\mathrm{E}}\right) \tau}-e^{-i\left(k_{n}+\mathbf{k} \cdot \mathbf{v}_{\mathrm{E}}\right) \tau}}{k_{0}-i k_{n}} \\
& \times\left\{\rho_{\mathrm{E}}\left(k_{0}, \mathbf{k}\right)\left[\frac{1}{\left(k_{n}+\mathbf{k} \cdot \mathbf{v}_{\mathrm{E}}\right)^{2}}\left(1+\frac{k_{n}^{2}}{k^{2}}\right)\right]\right. \\
& \left.+\rho_{\mathrm{T}}\left(k_{0}, \mathbf{k}\right)\left[\frac{r_{\perp}^{2}}{\left(\mathbf{k} \cdot \mathbf{r}_{\perp}\right)^{2}}+\frac{1}{\left(k_{n}+\mathbf{k} \cdot \mathbf{v}_{\mathrm{E}}\right)^{2}}\left(v_{\mathrm{E}}^{2}-\frac{k_{n}^{2}}{k^{2}}\right)\right]\right\}
\end{aligned}
$$

Here $k_{n} \equiv 2 \pi n / \beta$, with $n \in \mathbb{Z}$, are the Matsubara frequencies. The apparent poles of eq. (A.2) at $k_{n}+\mathbf{k} \cdot \mathbf{v}_{\mathrm{E}}=0$ are regulated by the zeros of the numerator.

The Matsubara sums can be carried out by partial fractioning the dependence on $k_{n}$, and then making use of

$$
\frac{1}{\beta} \sum_{k_{n}} \frac{e^{i k_{n} \tau}}{k_{0}-i k_{n}}=n_{\mathrm{B}}\left(k_{0}\right) e^{\tau k_{0}}, \quad \frac{1}{\beta} \sum_{k_{n}} \frac{e^{-i k_{n} \tau}}{k_{0}-i k_{n}}=n_{\mathrm{B}}\left(k_{0}\right) e^{(\beta-\tau) k_{0}}, \quad 0<\tau<\beta,
$$

where $n_{\mathrm{B}}\left(k_{0}\right) \equiv 1 /\left(e^{\beta k_{0}}-1\right)$. In order to simplify the expressions we also take the classical limit right away; recalling eq. (2.4) and setting $\hbar \rightarrow 0$, the results then become

$$
\begin{aligned}
C_{\mathrm{E}, \mathrm{cl}}^{(0)}\left(\tau, v_{\mathrm{E}}, r_{\perp}\right)=1, & g^{2} T C_{\mathrm{F}, \mathrm{cl}} \int_{\mathbf{k}}\left(\cos \mathbf{k} \cdot \mathbf{r}_{\perp}-1\right) \int_{-\infty}^{\infty} \frac{\mathrm{d} k_{0}}{\pi} \frac{2-e^{\left(k_{0}+i \mathbf{k} \cdot \mathbf{v}_{\mathrm{E}}\right) \tau}-e^{-\left(k_{0}+i \mathbf{k} \cdot \mathbf{v}_{\mathrm{E}}\right) \tau}}{k_{0}} \\
\times & \left\{\rho_{\mathrm{E}}\left(k_{0}, \mathbf{k}\right)\left[-\frac{1}{\left(k_{0}+i \mathbf{k} \cdot \mathbf{v}_{\mathrm{E}}\right)^{2}}\left(1-\frac{k_{0}^{2}}{k^{2}}\right)\right]\right. \\
& \left.+\rho_{\mathrm{T}}\left(k_{0}, \mathbf{k}\right)\left[\frac{r_{\perp}^{2}}{\left(\mathbf{k} \cdot \mathbf{r}_{\perp}\right)^{2}}-\frac{1}{\left(k_{0}+i \mathbf{k} \cdot \mathbf{v}_{\mathrm{E}}\right)^{2}}\left(v_{\mathrm{E}}^{2}+\frac{k_{0}^{2}}{k^{2}}\right)\right]\right\}
\end{aligned}
$$

It can be observed that in the classical limit, the Matsubara sum amounts effectively to replacing $k_{n}$ through $-i k_{0}$. (It would certainly be possible to keep $n_{\mathrm{B}}\left(k_{0}\right)$ in an exact form, cf. ref. [46] for $v=0$, however only the Bose-enhanced classical term $n_{\mathrm{B}}\left(k_{0}\right) \approx T /\left(\hbar k_{0}\right)$ is expected to contribute to the large- $t$ limit to be taken presently.) 
Wick rotation is carried out through $\tau=i t, v_{\mathrm{E}}=-i v$, and the potential is extracted from

$$
i \partial_{t} C_{\mathrm{E}, \mathrm{cl}}^{(2)}\left(i t,-i v, r_{\perp}\right) \equiv V_{\mathrm{cl}}^{(2)}\left(t, v, r_{\perp}\right) C_{\mathrm{E}, \mathrm{cl}}^{(0)}\left(i t,-i v, r_{\perp}\right)
$$

We obtain

$$
\begin{aligned}
V_{\mathrm{cl}}^{(2)}\left(t, v, r_{\perp}\right)= & g^{2} T C_{\mathrm{F}} \int_{\mathbf{k}}\left(\cos \mathbf{k} \cdot \mathbf{r}_{\perp}-1\right) \int_{-\infty}^{\infty} \frac{\mathrm{d} k_{0}}{\pi} \frac{e^{i\left(k_{0}+\mathbf{k} \cdot \mathbf{v}\right) t}-e^{-i\left(k_{0}+\mathbf{k} \cdot \mathbf{v}\right) t}}{k_{0}} \\
& \times\left\{\rho_{\mathrm{E}}\left(k_{0}, \mathbf{k}\right)\left[-\frac{1}{k_{0}+\mathbf{k} \cdot \mathbf{v}}\left(1-\frac{k_{0}^{2}}{k^{2}}\right)\right]\right. \\
& \left.+\rho_{\mathrm{T}}\left(k_{0}, \mathbf{k}\right)\left[\frac{r_{\perp}^{2}\left(k_{0}+\mathbf{k} \cdot \mathbf{v}\right)}{\left(\mathbf{k} \cdot \mathbf{r}_{\perp}\right)^{2}}+\frac{1}{k_{0}+\mathbf{k} \cdot \mathbf{v}}\left(v^{2}-\frac{k_{0}^{2}}{k^{2}}\right)\right]\right\} .
\end{aligned}
$$

Subsequently the large-time limit follows from

$$
\lim _{t \rightarrow \infty} \frac{e^{i\left(k_{0}+\mathbf{k} \cdot \mathbf{v}\right) t}-e^{-i\left(k_{0}+\mathbf{k} \cdot \mathbf{v}\right) t}}{k_{0}+\mathbf{k} \cdot \mathbf{v}}=2 \pi i \delta\left(k_{0}+\mathbf{k} \cdot \mathbf{v}\right) .
$$

Carrying out the integral over $k_{0}$ and setting also $v=1$, so that $\mathbf{k} \cdot \mathbf{v} \rightarrow k_{\|}$, leads to

$$
\begin{aligned}
V_{\mathrm{cl}}^{(2)}\left(\infty, 1, r_{\perp}\right)= & -i g^{2} T C_{\mathrm{F}} \int_{\mathbf{k}_{\perp}}\left(1-\cos \mathbf{k}_{\perp} \cdot \mathbf{r}_{\perp}\right) \\
& \times \int_{-\infty}^{\infty} \frac{\mathrm{d} k_{\|}}{\pi}\left\{\frac{\rho_{\mathrm{T}}\left(k_{\|}, \mathbf{k}\right)}{k_{\|}}-\frac{\rho_{\mathrm{E}}\left(k_{\|}, \mathbf{k}\right)}{k_{\|}}\right\} \frac{k_{\perp}^{2}}{k_{\perp}^{2}+k_{\|}^{2}} .
\end{aligned}
$$

Here we substituted $k_{\|} \rightarrow-k_{\|}$for simplicity. This potential is purely imaginary and, according to eq. (A.6), corresponds to an exponential decay of the light-cone Wilson loop at large Minkowskian times, as anticipated by eq. (1.1).

The next step is to perform the integral over $k_{\|}$. This is possible by re-expressing the spectral function as a discontinuity of the retarded correlator across the real axis,

$$
\rho\left(k_{0}, \mathbf{k}\right)=\frac{G_{\mathrm{R}}\left(k_{0}+i 0^{+}, \mathbf{k}\right)-G_{\mathrm{R}}\left(k_{0}-i 0^{+}, \mathbf{k}\right)}{2 i},
$$

and by then carrying out the contour integral. In the literature the procedure is known as a light-cone sum rule [14] (see also appendix A of ref. [13]), and yields

$$
\int_{-\infty}^{\infty} \frac{\mathrm{d} k_{\|}}{\pi} \frac{\rho\left(k_{\|}, \mathbf{k}\right)}{k_{\|}} \frac{k_{\perp}^{2}}{k_{\perp}^{2}+k_{\|}^{2}}=G_{\mathrm{R}}\left(0, \mathbf{k}_{\perp}\right)
$$

The retarded propagator is, in turn, the analytic continuation of the Euclidean one.

Recalling finally that the self-energy $\Pi_{\mathrm{T}}$ of eq. (3.1) vanishes at zero frequency, whereas $\Pi_{\mathrm{E}}$ equals the Debye mass parameter, $m_{\mathrm{E}}^{2}$, we recover eq. (3.5).

\section{B Leading-order perturbative computation on a lattice}

If the computation of appendix $\mathrm{A}$ is repeated in lattice regularization, then the expressions become a lot more complicated. For instance, employing the notation

$$
\tilde{k}_{i} \equiv \frac{2}{a} \sin \left(\frac{a k_{i}}{2}\right), \quad \underset{\sim}{k_{i}} \equiv \cos \left(\frac{a k_{i}}{2}\right), \quad \int_{\mathbf{k}} \equiv \int_{-\pi / a}^{\pi / a} \frac{\mathrm{d}^{3} \mathbf{k}}{(2 \pi)^{3}},
$$


and making use of Feynman rules derived from eq. (2.5), the observable of eq. (A.2) can formally be expressed as $\left(\mathbf{k}=\left(\mathbf{k}_{\perp}, k_{\|}\right), \mathbf{k}_{\perp} \equiv\left(k_{y}, k_{z}\right)\right)$

$$
\begin{aligned}
& C_{\mathrm{E}}^{(2)}\left(\tau, v_{\mathrm{E}}, r_{\perp}\right)=\frac{g_{0}^{2} C_{\mathrm{F}}}{\beta} \int_{\mathbf{k}}\left(\cos k_{y} r_{\perp}-1\right) \int_{-\infty}^{\infty} \frac{\mathrm{d} k_{0}}{\pi} \sum_{k_{n}} \frac{2-e^{i\left(k_{n}+k_{\|} v_{\mathrm{E}}\right) \tau}-e^{-i\left(k_{n}+k_{\|} v_{\mathrm{E}}\right) \tau}}{k_{0}-i k_{n}} \\
& \times\left\{\rho_{\mathrm{E}}\left(k_{0}, \mathbf{k}\right)\left(\underset{\sim}{k_{\|}}\right)^{2}\left(\widetilde{\frac{k_{n}}{v_{\mathrm{E}}}}\right)^{2}\left[\frac{1}{\left(\frac{k_{n}}{v_{\mathrm{E}}}+k_{\|}\right)^{2}}\left(\frac{1}{k_{n}^{2}}+\frac{1}{\tilde{k}^{2}}\right)\right]\right. \\
& \left.+\rho_{\mathrm{T}}\left(k_{0}, \mathbf{k}\right)\left[\frac{1}{\tilde{k}_{y}^{2}}+\frac{1}{\left(\frac{k_{n}}{v_{\mathrm{E}}}+k_{\|}\right)^{2}}\left(\left(\frac{k_{n}}{v_{\mathrm{E}}}\right)^{2}-\frac{1}{\tilde{k}^{2}}\left(\widetilde{\frac{k_{n}}{v_{\mathrm{E}}}}\right)^{2}\right)\right]\right\} \\
& -\frac{g_{0}^{2} C_{\mathrm{F}}}{\beta} \frac{\tau v_{\mathrm{E}} a^{3}}{4} \int_{\mathbf{k}} \int_{-\infty}^{\infty} \frac{\mathrm{d} k_{0}}{\pi} \sum_{k_{n}} \frac{1}{k_{0}-i k_{n}}\left(\frac{\widetilde{k_{n}}}{v_{\mathrm{E}}}\right)^{2} \\
& \times\left\{\rho_{\mathrm{E}}\left(k_{0}, \mathbf{k}\right)\left(\widetilde{k_{\|}}\right)^{2}\left(\frac{1}{k_{n}^{2}}+\frac{1}{\tilde{k}^{2}}\right)\right. \\
& \left.+\rho_{\mathrm{T}}\left(k_{0}, \mathbf{k}\right)\left(1-\frac{\left(\widetilde{k}_{\|}\right)^{2}}{\tilde{k}^{2}}\right)\right\} \text {. }
\end{aligned}
$$

If we recall, however, that after the Matsubara sum and the classical limit, $k_{n}$ gets essentially replaced by $-i k_{0}$, and that for non-zero distances and large times the contribution emerges from $k_{\perp} \lesssim m_{\mathrm{E}}$ and $k_{0}, k_{\|} \lesssim g^{2} T / \pi$ (cf. eq. (A.11)), then the lattice four-momenta can to a good approximation be replaced by their continuum limits,

$$
\tilde{k}_{\mu} \rightarrow k_{\mu}, \quad \underline{k}_{\mu} \rightarrow 1
$$

Then the first structure of eq. (B.2) goes over into eq. (A.2). In contrast, the second structure, which is linear in $\tau$ and independent of $r_{\perp}$, originates from self-energy corrections of the tilted Wilson lines and is specific to lattice regularization. Since this short-distance contribution arises from "hard" scales, there is no need for resummation; we can replace the spectral representations by free propagators,

$$
\int_{-\infty}^{\infty} \frac{\mathrm{d} k_{0}}{\pi} \frac{\rho_{\mathrm{T}(\mathrm{E})}(\mathcal{K})}{k_{0}-i k_{n}} \rightarrow \frac{1}{k_{n}^{2}+\tilde{k}^{2}} .
$$

Subsequently the Matsubara sum, classical limit, and analytic continuation are taken as usual, which ultimately leads to the intercept of eq. (3.9).

\section{References}

[1] CMS collaboration, Studies of jet quenching using isolated-photon+jet correlations in $\mathrm{PbPb}$ and pp collisions at $\sqrt{s_{N N}}=2.76$ TeV, Phys. Lett. B 718 (2013) 773 [arXiv:1205.0206] [INSPIRE].

[2] R. Baier, D. Schiff and B.G. Zakharov, Energy loss in perturbative QCD, Ann. Rev. Nucl. Part. Sci. 50 (2000) 37 [hep-ph/0002198] [InSPIRE].

[3] J. Casalderrey-Solana and C.A. Salgado, Introductory lectures on jet quenching in heavy ion collisions, Acta Phys. Polon. B 38 (2007) 3731 [arXiv:0712.3443] [INSPIRE]. 
[4] U.A. Wiedemann, Jet Quenching in Heavy Ion Collisions, arXiv:0908.2306 [INSPIRE].

[5] A. Majumder and M. van Leeuwen, The Theory and Phenomenology of Perturbative QCD Based Jet Quenching, Prog. Part. Nucl. Phys. A 66 (2011) 41 [arXiv:1002.2206] [InSPIRE].

[6] N. Armesto, B. Cole, C. Gale, W.A. Horowitz, P. Jacobs et al., Comparison of jet quenching formalisms for a quark-gluon plasma 'brick', Phys. Rev. C 86 (2012) 064904 [arXiv:1106.1106] [INSPIRE].

[7] F. D'Eramo, M. Lekaveckas, H. Liu and K. Rajagopal, Momentum broadening in weakly coupled quark-gluon plasma (with a view to finding the quasiparticles within liquid quark-gluon plasma), JHEP 05 (2013) 031 [arXiv:1211.1922] [INSPIRE].

[8] Y. Mehtar-Tani, J. Milhano and K. Tywoniuk, Jet physics in heavy-ion collisions, Int. J. Mod. Phys. A 28 (2013) 1340013 [arXiv:1302.2579] [INSPIRE].

[9] J. Casalderrey-Solana and D. Teaney, Transverse Momentum Broadening of a Fast Quark in $a \mathcal{N}=4$ Yang-Mills Plasma, JHEP 04 (2007) 039 [hep-th/0701123] [INSPIRE].

[10] Z.-t. Liang, X.-N. Wang and J. Zhou, The Transverse-momentum-dependent Parton Distribution Function and Jet Transport in Medium, Phys. Rev. D 77 (2008) 125010 [arXiv:0801.0434] [INSPIRE].

[11] F. D'Eramo, H. Liu and K. Rajagopal, Transverse Momentum Broadening and the Jet Quenching Parameter, Redux, Phys. Rev. D 84 (2011) 065015 [arXiv:1006.1367] [INSPIRE].

[12] M. Benzke, N. Brambilla, M.A. Escobedo and A. Vairo, Gauge invariant definition of the jet quenching parameter, JHEP 02 (2013) 129 [arXiv: 1208.4253] [INSPIRE].

[13] S. Caron-Huot, O(g) plasma effects in jet quenching, Phys. Rev. D 79 (2009) 065039 [arXiv: 0811.1603] [INSPIRE].

[14] P. Aurenche, F. Gelis and H. Zaraket, A Simple sum rule for the thermal gluon spectral function and applications, JHEP 05 (2002) 043 [hep-ph/0204146] [INSPIRE].

[15] M. Laine, A non-perturbative contribution to jet quenching, Eur. Phys. J. C 72 (2012) 2233 [arXiv: 1208.5707] [INSPIRE].

[16] D.Y. Grigoriev and V. Rubakov, Soliton pair creation at finite temperatures. Numerical study in (1+1)-dimensions, Nucl. Phys. B 299 (1988) 67 [INSPIRE].

[17] J. Ambjørn, T. Askgaard, H. Porter and M.E. Shaposhnikov, Sphaleron transitions and baryon asymmetry: A Numerical real time analysis, Nucl. Phys. B 353 (1991) 346 [INSPIRE].

[18] J. Ambjørn and A. Krasnitz, Improved determination of the classical sphaleron transition rate, Nucl. Phys. B 506 (1997) 387 [hep-ph/9705380] [INSPIRE].

[19] G.D. Moore and K. Rummukainen, Classical sphaleron rate on fine lattices, Phys. Rev. D 61 (2000) 105008 [hep-ph/9906259] [INSPIRE].

[20] G.D. Moore and M. Tassler, The Sphaleron Rate in $\mathrm{SU}(N)$ Gauge Theory, JHEP 02 (2011) 105 [arXiv: 1011.1167] [INSPIRE].

[21] M. D'Onofrio, K. Rummukainen and A. Tranberg, The Sphaleron Rate through the Electroweak Cross-over, JHEP 08 (2012) 123 [arXiv:1207.0685] [INSPIRE].

[22] M. Hindmarsh and A. Rajantie, Phase transition dynamics in the hot Abelian Higgs model, Phys. Rev. D 64 (2001) 065016 [hep-ph/0103311] [INSPIRE]. 
[23] D. Bödeker, L.D. McLerran and A.V. Smilga, Really computing nonperturbative real time correlation functions, Phys. Rev. D 52 (1995) 4675 [hep-th/9504123] [INSPIRE].

[24] P.B. Arnold, Hot B violation, the lattice and hard thermal loops, Phys. Rev. D 55 (1997) 7781 [hep-ph/9701393] [INSPIRE].

[25] M. Laine, O. Philipsen and M. Tassler, Thermal imaginary part of a real-time static potential from classical lattice gauge theory simulations, JHEP 09 (2007) 066 [arXiv:0707.2458] [INSPIRE].

[26] M. Laine, G.D. Moore, O. Philipsen and M. Tassler, Heavy Quark Thermalization in Classical Lattice Gauge Theory: Lessons for Strongly-Coupled QCD, JHEP 05 (2009) 014 [arXiv: 0902.2856] [INSPIRE].

[27] A. Rothkopf, T. Hatsuda and S. Sasaki, Complex Heavy-Quark Potential at Finite Temperature from Lattice QCD, Phys. Rev. Lett. 108 (2012) 162001 [arXiv:1108.1579] [INSPIRE].

[28] S. Caron-Huot, M. Laine and G.D. Moore, A Way to estimate the heavy quark thermalization rate from the lattice, JHEP 04 (2009) 053 [arXiv: 0901.1195] [INSPIRE].

[29] H.B. Meyer, The errant life of a heavy quark in the quark-gluon plasma, New J. Phys. 13 (2011) 035008 [arXiv:1012.0234] [InSPIRE].

[30] A. Francis, O. Kaczmarek, M. Laine and J. Langelage, Towards a non-perturbative measurement of the heavy quark momentum diffusion coefficient, PoS LATTICE 2011 (2011) 202 [arXiv: 1109.3941] [INSPIRE].

[31] D. Banerjee, S. Datta, R. Gavai and P. Majumdar, Heavy Quark Momentum Diffusion Coefficient from Lattice QCD, Phys. Rev. D 85 (2012) 014510 [arXiv:1109.5738] [INSPIRE].

[32] B. Schenke, M. Strickland, A. Dumitru, Y. Nara and C. Greiner, Transverse momentum diffusion and jet energy loss in non-Abelian plasmas, Phys. Rev. C 79 (2009) 034903 [arXiv:0810.1314] [INSPIRE].

[33] M. Laine, O. Philipsen, P. Romatschke and M. Tassler, Real-time static potential in hot QCD, JHEP 03 (2007) 054 [hep-ph/0611300] [inSPIRE].

[34] L. Giusti and H.B. Meyer, Implications of Poincaré symmetry for thermal field theories in finite-volume, JHEP 01 (2013) 140 [arXiv:1211.6669] [INSPIRE].

[35] D. Bödeker, Classical real time correlation functions and quantum corrections at finite temperature, Nucl. Phys. B 486 (1997) 500 [hep-th/9609170] [INSPIRE].

[36] J.B. Kogut and L. Susskind, Hamiltonian Formulation of Wilson's Lattice Gauge Theories, Phys. Rev. D 11 (1975) 395 [InSPIRE].

[37] J. Frenkel and J.C. Taylor, Hard thermal QCD, forward scattering and effective actions, Nucl. Phys. B 374 (1992) 156 [inSPIRE].

[38] E. Braaten and R.D. Pisarski, Simple effective Lagrangian for hard thermal loops, Phys. Rev. D 45 (1992) 1827 [inSPIRE].

[39] G.N. Watson, Three triple integrals, Q. J. Math. 10 (1939) 266.

[40] M.L. Glasser and J. Boersma, Exact values for the cubic lattice Green functions, J. Phys. A: Math. Gen. 33 (2000) 5017.

[41] B. Lucini and M. Teper, $\mathrm{SU}(N)$ gauge theories in (2+1)-dimensions: Further results, Phys. Rev. D 66 (2002) 097502 [hep-lat/0206027] [INSPIRE]. 
[42] P.H. Ginsparg, First Order and Second Order Phase Transitions in Gauge Theories at Finite Temperature, Nucl. Phys. B 170 (1980) 388 [inSPIRE].

[43] T. Appelquist and R.D. Pisarski, High-Temperature Yang-Mills Theories and Three-Dimensional Quantum Chromodynamics, Phys. Rev. D 23 (1981) 2305 [InSPIRE].

[44] Y. Burnier and A. Rothkopf, Disentangling the timescales behind the non-perturbative heavy quark potential, Phys. Rev. D 86 (2012) 051503 [arXiv: 1208.1899] [INSPIRE].

[45] Y. Burnier and A. Rothkopf, A hard thermal loop benchmark for the extraction of the nonperturbative $Q \bar{Q}$ potential, arXiv:1304.4154 [INSPIRE].

[46] M. Laine, A Resummed perturbative estimate for the quarkonium spectral function in hot QCD, JHEP 05 (2007) 028 [arXiv:0704.1720] [INSPIRE]. 